\title{
Robust operation of copper chemical mechanical polishing
}

\author{
Yi-Chung Kao ${ }^{\mathrm{a}}$, Cheng-Ching $\mathrm{Yu}^{\mathrm{b}}{ }^{*}$, Shih-Haur Shen ${ }^{\mathrm{c}}$ \\ ${ }^{a}$ Department of Chemical Engineering, National Taiwan University Science Technology, Taipei 106-07, Taiwan \\ ${ }^{\mathrm{b}}$ Department of Chemical Engineering, National Taiwan University, Taipei 106-17, Taiwan \\ ${ }^{\mathrm{c}}$ Applied Materials Taiwan, Hsin-Chu 300, Taiwan
}

Received 3 December 2001; accepted 19 March 2002

\begin{abstract}
The unusual concentration effect in copper chemical mechanical polishing (CMP) is verified experimentally and operation-relevant models are derived. First, the well-known Preston equation is modified by taking the concentration effects into account. Next, a linear model is proposed to describe dishing as functions of and typical operation variables. Combining both models, we are able to locate feasible operation region without constraint violation. Finally, the robust operation procedure is incorporated into a realistic CMP operation strategy: soft landing. A systematic procedure is proposed to ensure robust operation for copper CMP.
\end{abstract}

(C) 2003 Elsevier Science B.V. All rights reserved.

Keywords: Chemical mechanical polishing; Copper CMP; Removal rate; Dishing; Process control

\section{Introduction}

The complexity of current microelectronic devices demands global planarity at different metallization levels. Chemical mechanical polishing (CMP) has the capability of achieving such stringent requirements over a step height of several microns. Since the introduction of the new technology in the 1980's, the CMP provides advantages of defect reduction, wide windows for etching and lithography and yield improvement $[3,7]$. Combining the chemical reactions and mechanical force abrasion, the wafer surface can be polished to achieve global planarity. Despite recent advances in CMP, some manufacturing concerns associated with successful implementation of CMP remain to be overcome $[1,3,7]$. The physical interactions among the wafer, slurry, and pad make the copper CMP very sensitive to operating conditions. If the CMP is operated in an undesirable region, it may lead to non-uniformity, surface damage and/or degradation in the removal rate (RR). This is especially true

\footnotetext{
*Corresponding author. Fax: + 886-2-2362-3040.

E-mail address: ccyu@ccms.ntu.edu.tw (C.-C. Yu).
} 
for the copper CMP with hydrogen peroxide $\left(\mathrm{H}_{2} \mathrm{O}_{2}\right)$-based slurry, because the removal rate is a non-monotonic function of the oxidizer $\left(\mathrm{H}_{2} \mathrm{O}_{2}\right)$ concentration [8,10]. This may lead to severe manufacturing problems, because, during operation, the concentration of the oxidizer decreases gradually, and, if the process is operated at a concentration sensitive region, a significant variation in the RR may result. Therefore, it is important to find an appropriate region such that stable operation can be achieved. Typical operating variables in a CMP system include: oxidizer concentration in the slurry, slurry flow rate, platen and polishing heads rotation speeds, and down force (pressure). The objective of this work is to devise a systematic procedure to ensure robust operation. The remainder of this paper is organized as follows. Experimental results describing the oxidizer concentration effects are presented and interpretations are given in Section 2. Operation-relevant models, addressing concentration effect and dishing, are constructed in Section 3. In Section 4, a procedure to locate a feasible operation region is presented and an overall strategy to ensure robust operation while maintaining high throughput is proposed. Conclusions are drawn in Section 5.

\section{Experimental}

\subsection{Experimental set-up}

Copper $(\mathrm{Cu}) \mathrm{CMP}$ was carried out on a Applied Materials' Mirra ${ }^{\mathrm{TM}}$ polisher using Titan ${ }^{\mathrm{TM}}$ polishing heads. Polyurethane pads were used and the experimental copper CMP slurry with alumina abrasive particles were provided by Cabot Corporation. A given amount of hydrogen peroxide oxidizer was added to the slurry to achieve a specified concentration (wt.\%) and it is well mixed prior to the polishing. The slurry was continuously agitated during the experiments. The wafer pressure $(P)$ was varied between 2 to 4 p.s.i., the platen speed $(V)$ was changed from 63 to $93 \mathrm{rpm}$ (round per minute) and the corresponding head speeds are 65 and $95 \mathrm{rpm}$, respectively. The slurry flow rate was set to $200 \mathrm{ml} / \mathrm{min}$. In each case, the endpoint was detected with the ISRM $^{\mathrm{TM}}$ system.

Copper deposition was carried out with the physical vapor deposition (PVD) process for barrier $(\mathrm{TaN})$ and $\mathrm{Cu}$ seed. Electrochemical plating (ECP) process is employed for $\mathrm{Cu}$ plating. All copper thickness measurements were performed with a Tencor RS75 and the erosion and dishing measurements were performed with a Tencor HRP200 high-resolution profilometer.

\subsection{Results and interpretation}

The experiments were carried out over a wide range of oxidizer concentration (0.5-15 wt.\%) and for two different pressures and speeds. Fig. 1 clearly indicates that, as the oxidizer concentration increases, the removal rate goes up initially followed by a gradual decay and the trend holds for different combinations of pressure and speed.

Similar behavior was observed in aluminum (Al) CMP and Cu CMP using $\mathrm{H}_{2} \mathrm{O}_{2}$-based slurry and explanations are also given [8,10]. Basically, the interpretation follows the planarization mechanism proposed by Kaufman et al. [2]. At the region of low oxidizer concentration, the rate of oxide generation is small and the passivation layer is removed as soon as it is formed (Region 1 in Fig. 2). In other words, region 1 is a chemical reaction controlled process. Then an equilibrium is reached when the rate of passivation is equal to the rate of mechanical abrasion. This corresponds to the 


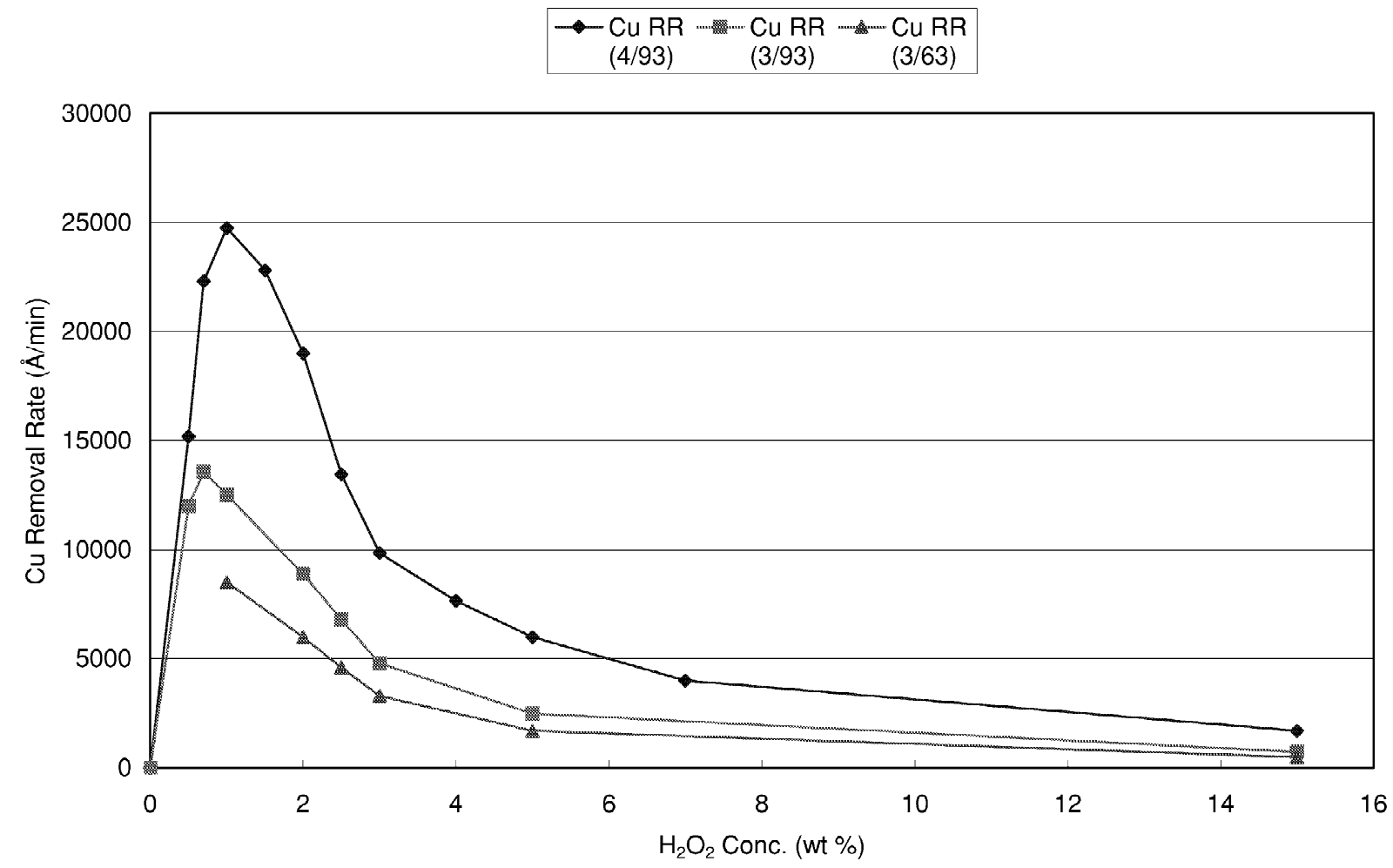

Fig. 1. Removal rate $(\AA / \mathrm{min})$ as a function of oxidizer concentration for different pressure and speed settings: $P=4$ p.s.i. and $V=93 \mathrm{rpm}, P=3$ p.s.i. and $V=93 \mathrm{rpm}$, and $P=3$ p.s.i. and $V=63 \mathrm{rpm}$.

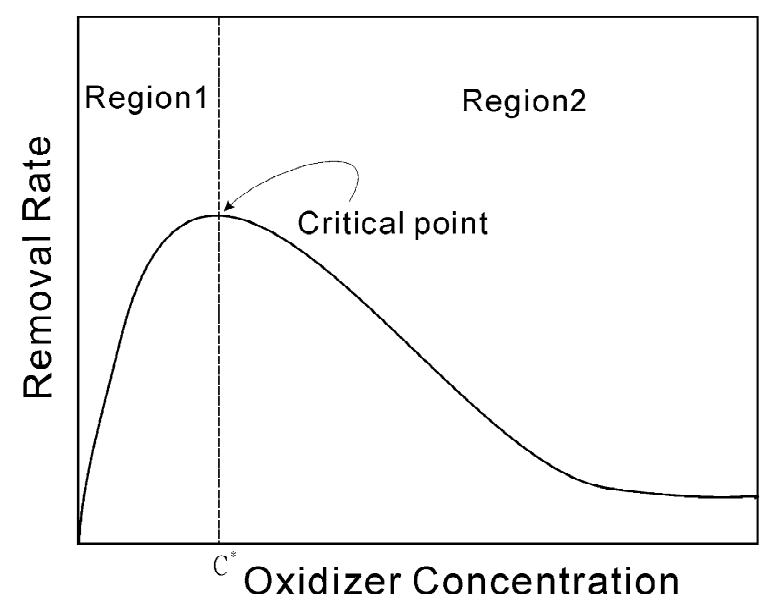

Fig. 2. Effects of oxidizer concentration on removal rate (Region 1: chemical reaction controlled and Region 2: mechanical abrasion controlled). 
maximal removal rate (Figs. 1 and 2). As we increase the oxide concentration further, not only a passivation layer is formed, but the passivation itself changes its structure (e.g. strength, diffusion property etc. $[5,10])$. This creates a barrier for mechanical abrasion and slows down the removal rate (Region 2 in Fig. 2). Despite the fact that different mechanisms are proposed, similar behaviors are observed for different metal CMP (tungsten, aluminum, and copper) using $\mathrm{H}_{2} \mathrm{O}_{2}$-based slurry. Moreover, this makes the already very difficult operation problem even worse. A question naturally arises: what is an appropriate oxidizer concentration?

\section{Operation-relevant models}

In process operation, we would like to achieve a certain removal rate while maintaining uniformity and minimizing possible surface damage (e.g. dishing, erosion etc.) under uncertainties (e.g. slow decay in slurry chemistry, defect in the polish pad etc.). Predictive models are useful to locate feasible operation region for adjustable process parameters. The handles we have are: pressure $(P)$, speed $(V)$ and oxidizer concentration $(C)$. Two types of models are of practical importance. One is a model describing the RR as a function of $P, V$, and $C$, and the other is a model relating dishing to $P$ and $V$.

\subsection{Effects of oxidizer concentration}

\subsubsection{Region 1}

From the experimental results (Fig. 1), it is clear that region 1 is not a feasible operation region, since a small change in the oxidizer concentration can lead to a significant change in RR. As mentioned earlier, this is a chemical reaction controlled process and it can be approximated reasonably with a straight line. This corresponds to a first order reaction kinetics and we have:

$$
\mathrm{RR}=k \cdot C
$$

where RR is the removal rate $(\AA / \mathrm{min}), k$ is the rate constant and $C$ is the oxidizer concentration (wt.\%). The least square regression of region 1 data gives $k=25600$.

\subsubsection{Region 2}

This is the region where most $\mathrm{Cu}$ CMP is carried out. It describes the change of properties in the passivating layer and thus hinders the removal rate. The experimental results (Fig. 1) reveal that, for various pressure and speed combinations, the RR decays exponentially. Before taking the concentration effect into account, we need a fundamental equation to represent the RR. The Preston equation [6] probably is the most popular equation to describe removal (polishing) rate. The success of the equation comes from its simplicity and links to measurable (computable) variables. For a generalized version, the removal rate $(\mathrm{RR})$ can be expressed as:

$$
\mathrm{RR}=K_{\mathrm{p}} P^{\alpha} V^{\beta}
$$

where $K_{\mathrm{p}}$ is the Preston constant, $\alpha$ and $\beta$ are exponents for the pressure and speed, respectively, and, in the original Preston expression, we have $\alpha=\beta=1$. Cook [3,4] further relates the Preston constant to the Young's modulus in the following way: 


$$
K_{\mathrm{p}}=\frac{1}{2 E}
$$

where $E$ is the Young's modulus of the material removed. As pointed out earlier, in region 2, the passivation itself changes its structure [10] as the concentration increases. Therefore, the Preston coefficient can be expressed as a function of the oxidizer concentration. Here, we use a linear mixing rule to show the characteristic of changing property. Two distinct states are defined first and a linear combination is used to describe the gradual transition.

$$
K_{\mathrm{p}}=x K_{\mathrm{p}, 1}+(1-x) K_{\mathrm{p}, 2}
$$

where $K_{\mathrm{p}, 1}$ is the Preston constant at equilibrium point (showing the maximum RR, $\mathrm{RR}_{\max }$, in Fig. 2), $K_{\mathrm{p}, 2}$ represents the Preston constant when the oxidizer concentration becomes extremely large and it corresponds to the minimum removal rate, $\mathrm{RR}_{\min }$. The concentration effect is described by $x$ indicating how fast the RR changes with concentration. We assume that the RR decay exponentially and this gives:

$$
x=\mathrm{e}^{-\frac{C-C^{*}}{C_{\mathrm{o}}}}
$$

where $C^{*}$ is the concentration giving the maximum RR (Fig. 2) and $C_{\mathrm{o}}$ describes the speed of decay in the RR. Combining Eqs. (4) and (5), we obtain an equation taking the concentration effect into account.

$$
\mathrm{RR}=\mathrm{e}^{-\frac{C-C^{*}}{C_{\mathrm{o}}}} \mathrm{RR}_{\max }+\left(1-\mathrm{e}^{-\frac{C-C^{*}}{C_{\mathrm{o}}}}\right) \mathrm{RR}_{\min }
$$

Once the model structure is set, we can find the exponents of the Preston equation (Eq. (2)). The experimental data (Fig. 1) show that, in region 2, the removal rate varies almost linearly with speed $(V)$. Therefore, we have $\beta=1$ and the only exponent left is $\alpha$. Taking the logarithm, the regression becomes a linear least square problem.

$$
\ln (\mathrm{RR})-\ln (V)=\ln \left(K_{\mathrm{p}}\right)+\alpha \ln (P)
$$

Using the data at $C=2.5 \%$ and assuming a constant $K_{\mathrm{p}}$, one obtains $\alpha=2.375$. Thus, we have the following modified Preston equation:

$$
\mathrm{RR}=K_{\mathrm{p}} P^{2.375} V
$$

Combining with the concentration effect, the equation becomes:

$$
\mathrm{RR}=\mathrm{e}^{-\frac{C-C^{*}}{C_{\mathrm{o}}}} K_{\mathrm{p}, 1} P^{2.375} V+\left(1-\mathrm{e}^{-\frac{C-C^{*}}{C_{\mathrm{o}}}}\right) K_{\mathrm{p}, 2} P^{2.375} V
$$

Non-linear regression was carried out for each set of experimental data and the model parameters for each data set are shown in Table 1 . The results of using averaged model parameters (model 4) and individual set of model parameters (models 1-3), optimal model parameters, are compared. Fig. 3 indicates that good results can be maintained by assuming constant $K_{\mathrm{p}, 1}, K_{\mathrm{p}, 2}$ and $C_{\mathrm{o}}$. In other words, the same model parameters $\left(K_{\mathrm{p}, 1}, K_{\mathrm{p}, 2}\right.$ and $\left.C_{\mathrm{o}}\right)$ can be used at different operation conditions (i.e. different $P$ and $V$ ). 
Table 1

Model parameters from the regression of individual data set

\begin{tabular}{|c|c|c|c|}
\hline & $\begin{array}{l}\text { Pressure }=3 \text { (p.s.i. }) \\
\text { Speed }=63(\mathrm{rpm})\end{array}$ & $\begin{array}{l}\text { Pressure = } 3 \text { (p.s.i.) } \\
\text { Speed =93 (rpm) }\end{array}$ & $\begin{array}{l}\text { Pressure }=4 \text { (p.s.i. }) \\
\text { Speed }=93(\mathrm{rpm})\end{array}$ \\
\hline$\overline{C_{\mathrm{o}}}$ & 2.63 & 2.43 & 2.40 \\
\hline $\mathrm{RR}_{\min }(\AA / \mathrm{min})$ & 261 & 521 & 1516 \\
\hline$K_{\mathrm{p}, 1}$ & 11.87 & 10.75 & 9.88 \\
\hline$K_{\mathrm{p}, 2}^{\mathrm{p}, 1}$ & 0.31 & 0.41 & 0.61 \\
\hline
\end{tabular}

\subsection{Modeling dishing}

The experimental data of Wijekoon et al. [9] provide useful insight in modeling dishing. Fig. 4 indicates that at a constant speed (or pressure) the dishing $(D)$ varies linearly with pressure (or speed). Therefore, a multilinear model can be constructed to describe dishing.
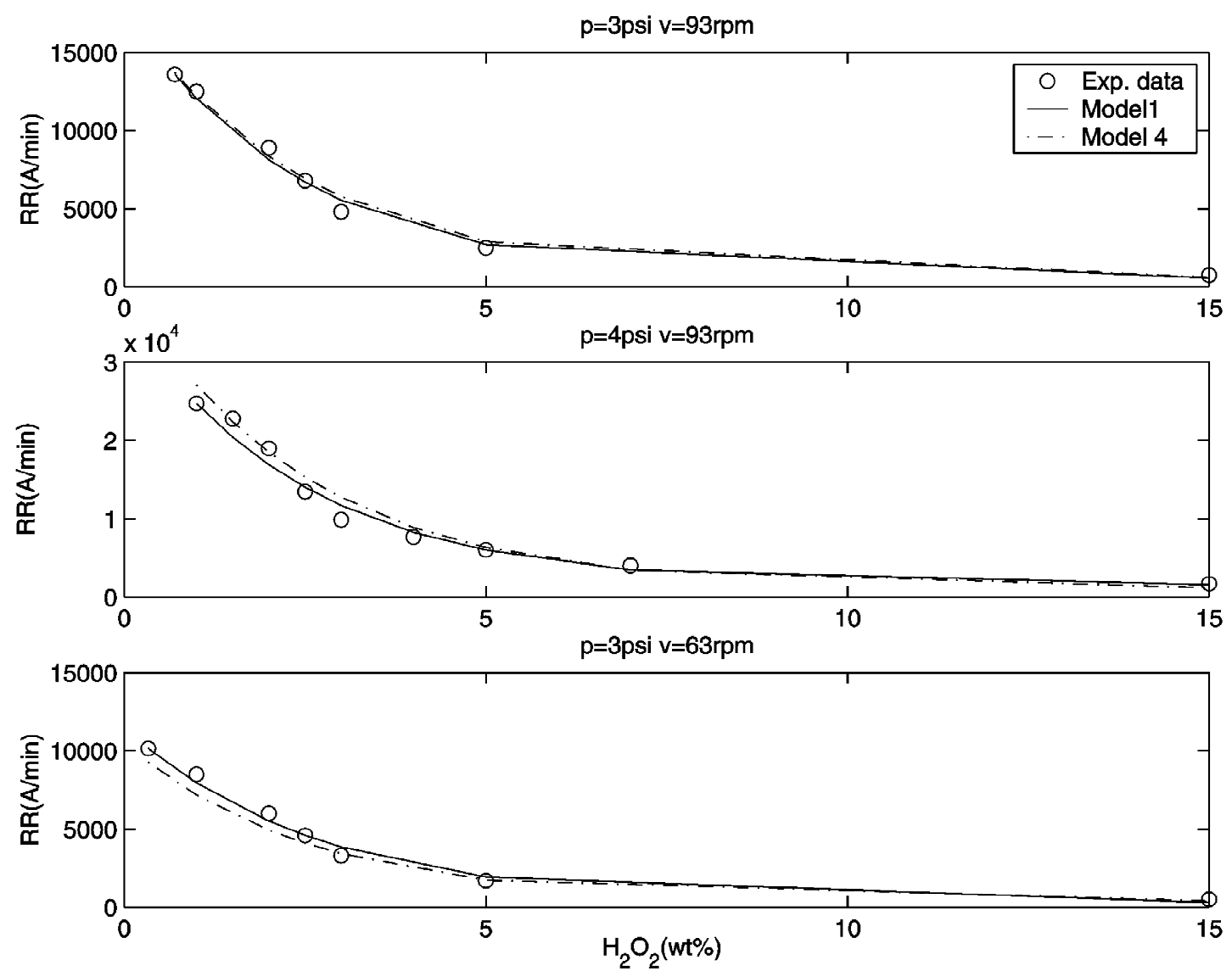

Fig. 3. Regression results using parameters for individual data set (model 1) and averaged model parameters (model 4). 

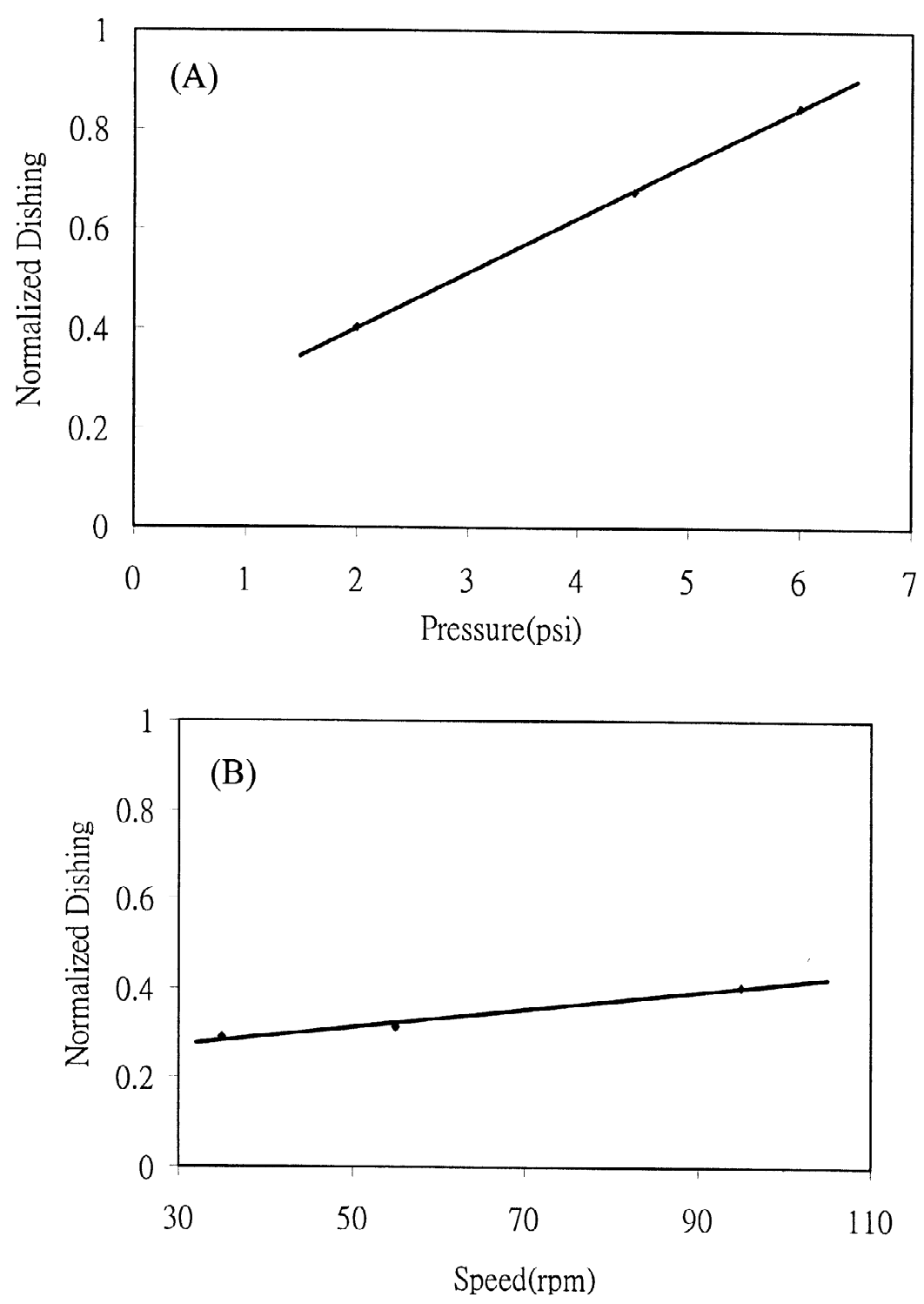

Fig. 4. Effect of pressure and speed on dishing (Wijekoon et al., 1998).

$$
D=a_{0}+a_{1} P+a_{2} V
$$

where $D$ is the dishing in a normalized unit, $P$ is the pressure in p.s.i. and $V$ is the speed in rpm. The linear least square regression of the data (Fig. 4) gives:

$$
D=-0.007+0.110 P+0.002 \mathrm{~V}
$$

As shown in the experimental data (or Eq. (11)), the pressure has a much larger effect on dishing, 
compared to the speed. Eq. (11) clearly describes the impacts of process variables on the important operation issue, dishing.

\section{Robust operation}

\subsection{Operation concerns}

Two operation related problems are addressed here. Dishing of copper lines is one of the most important issues in $\mathrm{Cu}$ CMP. Dishing reduces the final thickness of the copper lines and degrades the planarity of the wafer surface. Fig. 4 clearly indicates that an increase in the pressure or speed leads to an increase in the dishing and, moreover, the pressure has a larger effect on dishing than the speed (e.g. Eq. (11)). Therefore, even with the same RR, we have an extra degree of freedom, by adjusting $P$ and $V$, to minimize dishing. A typical constraint on dishing is: $D \leq 0.5$. The next problem is the variation in the removal rate as a result of oxidizer concentration changes. Fig. 1 shows that, around the region of $\mathrm{RR}_{\max }$, a small change in the oxidizer concentration may lead to drastically different $\mathrm{RR}$. We would like to avoid operating CMP around this sensitive region, because the oxidizer concentration generally decays with time. The sensitivity of RR with respect to $C$ can be derived directly from Eq. (9) by taking the derivative.

$$
S \equiv\left|\frac{\mathrm{dRR}}{\mathrm{dC}}\right|=\left(K_{\mathrm{p}, 1}-K_{\mathrm{p}, 2}\right) P^{\alpha} V\left(\frac{\mathrm{e}^{-\left(C-C^{*}\right) / C_{\mathrm{o}}}}{C_{\mathrm{o}}}\right)
$$

The sensitivity is denoted as $S$ hereafter. Eq. (12) reveals that a large pressure or speed leads to higher sensitivity and it also indicates that a large oxidizer concentration reduces the sensitivity. Fig. 5 shows the effects of pressure and oxidizer concentration on the sensitivity. A typical upper limit for the sensitivity is $2000(\AA / \mathrm{min} / \mathrm{wt} . \%)$ and this means that, at most, $1000 \AA / \mathrm{min}$ variation in RR is permitted for a $0.5 \mathrm{wt} \%$ oxidizer concentration change.

\subsection{Operation region}

\subsubsection{Feasible operation region}

Ongoing analyses indicate that we have three operation-relevant equations (Eqs. (9), (11) and (12)). One (Eq. (9)) relates process specification (RR) to operation variables $(P, V$, and $C$ ) and the other two (Eqs. (11) and (12)) describe process constraints (e.g. dishing and sensitivity) in terms of $P, V$, and $C$. In theory, a large number of $P-V-C$ combinations can achieve a certain removal rate, but, in practice, constraints are often imposed. As pointed out earlier, major operation concerns lead to the following constraints on dishing and sensitivity: $D \leq 0.5$ and $S \leq 2000$. With constraints inactive, we are able to locate feasible operation regions. For example, for $\mathrm{RR}=2000 \AA / \mathrm{min}$, we can obtain the entire region by solving Eq. (9) subject to the following constraints:

$$
\begin{aligned}
& D=-0.007+0.110 P+0.002 V \leq 0.5 \\
& |\mathrm{dRR} / \mathrm{d} C|=\left(K_{\mathrm{p}, 1}-K_{\mathrm{p}, 2}\right) P^{\alpha} V\left(\frac{\mathrm{e}^{-\left(C-C^{*}\right) / C_{\mathrm{o}}}}{C_{\mathrm{o}}}\right) \leq 2000
\end{aligned}
$$




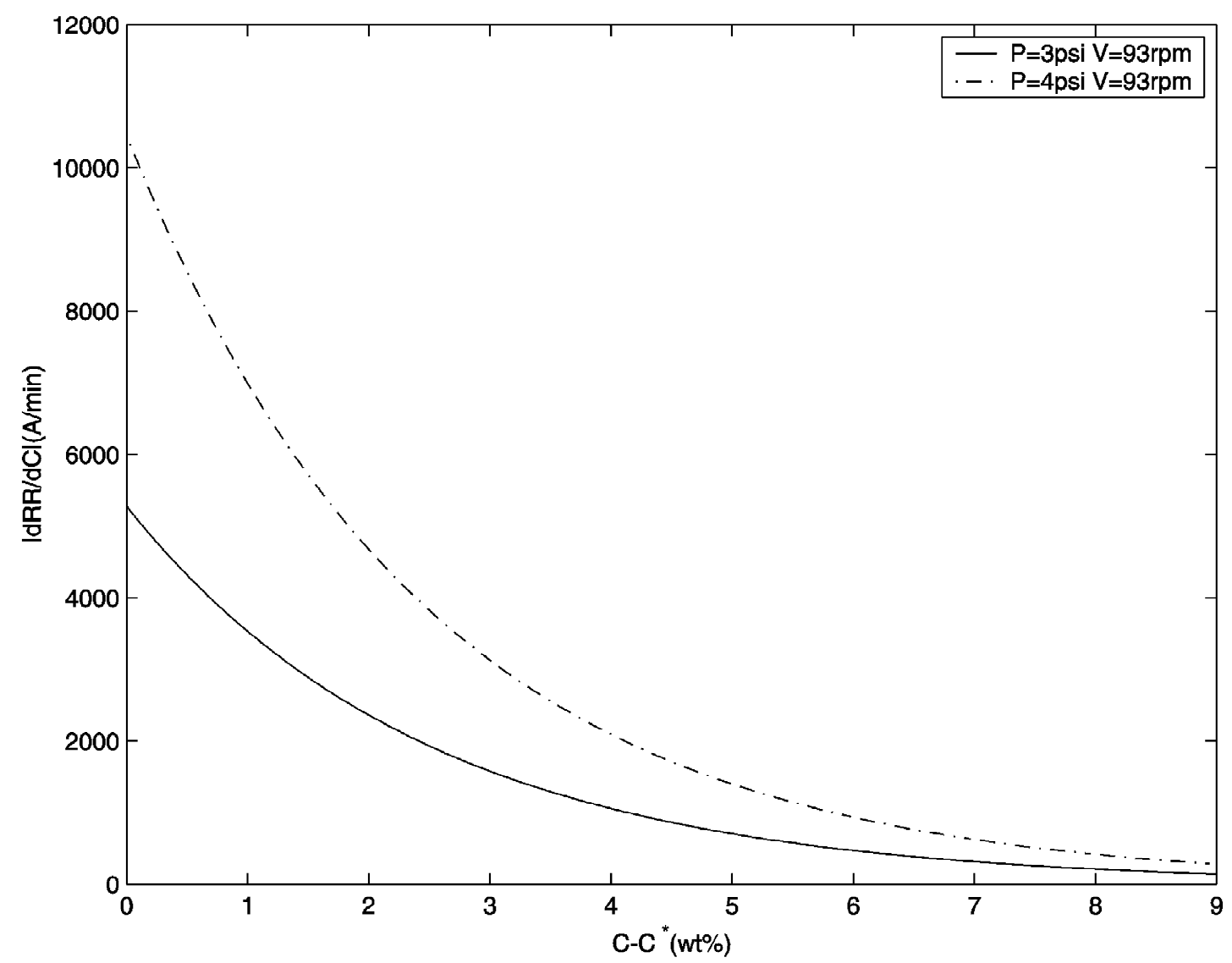

Fig. 5. Sensitivity as a function of oxidizer concentration.

Fig. 6 shows that, for $R R=2000$, the feasible region is a surface with pressure ranging from 1.5 to 4 p.s.i., speed ranging from 40 to $140 \mathrm{rpm}$, and oxidizer concentration changing 0.08 to 5.9 wt.\%. However, as a higher removal rate is required (e.g. $\mathrm{RR}=5000 \AA / \mathrm{min}$ ), the feasible operation region is much smaller, e.g. oxidizer concentration changing from 0.2 to $3.1 \mathrm{wt} \%$, as shown in Fig. 7.

\subsubsection{Optimized operation condition}

From all possible operation points in the feasible region (Figs. 6 and 7), one can select a certain objective function for further optimization and, in doing so, we can set operation variables $(P, V$ and $C$ ) for $\mathrm{Cu} \mathrm{CMP}$. One obvious objective is to minimize dishing. This can be derived analytically by substituting Eqs. (9) and (12) into Eq. (11) and taking the derivative of $D$ with respect to $P$. After some algebraic manipulation, one obtains:

$$
P=\left\{\frac{0.002 \alpha\left(\mathrm{RR}-\left|\frac{\mathrm{dRR}}{\mathrm{d} C}\right| C_{0}\right)}{0.110 K_{\mathrm{p}, 2}}\right\}^{\frac{1}{1+\alpha}}
$$

Eq. (15) clearly indicates that a large $S(|\mathrm{dRR} / \mathrm{d} C|)$ gives small $P$ and this, subsequently, leads to a 


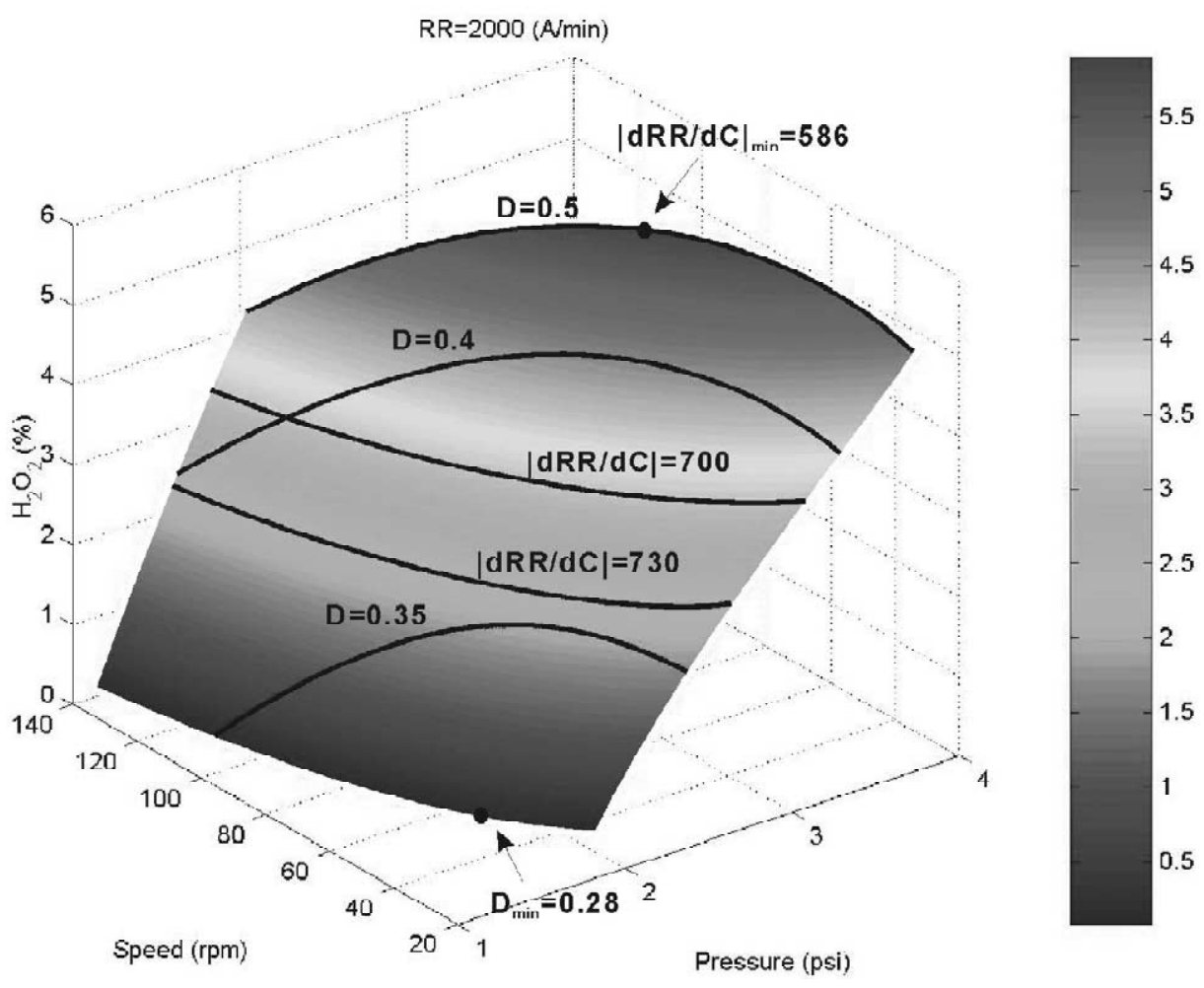

Fig. 6. Feasible operation region $(D \leq 0.5$ and $S \leq 2000)$ and constant dishing and sensitivity contours for RR=2000 $\AA / \min$.

minimal dishing. As shown in Fig. 5, the largest $S$ corresponds to the smallest oxidizer concentration. Therefore, a simple way to minimize dishing is set the oxidizer concentration to the smallest allowable value. Figs. 6 and 7 show that the operation condition with minimal dishing $\left(D_{\text {min }}\right)$ corresponds to the smallest $C$ (or the largest $S$ ) and numerical values for $\mathrm{RR}=2000$ and $5000 \AA / \mathrm{min}$ are given in Tables 2 and 3. On the contrary, if we choose to minimize the sensitivity, the results show that the oxidizer concentration should be set to the largest value (Figs. 6 and 7) and this corresponds to the upper limit of dishing. The results reveal that the two optimized operation conditions $\left(D_{\min }\right.$ and $\left.|\mathrm{dRR} / \mathrm{d} C|_{\min }\right)$ lie on the opposite sides of the feasible operation region (the smallest $C$ and the largest $C$ ). Moreover, as opposed to one's intuition, the oxidizer concentration plays an important role in setting the operation condition.

The optimized results in Tables 2 and 3 also reveal that, for the case of minimized dishing, the oxidizer concentration becomes unreasonably small (much less than $1 \mathrm{wt} \%$ ) and this may raise problems in practice. The reason is that, for a polisher to work properly, there are certain criteria to be met. Typical operable ranges for $\mathrm{Cu} C \mathrm{CMP}$ are: $P \geq 1$ p.s.i., $V \geq 40 \mathrm{rpm}$, and $C \geq 2 \mathrm{wt}$. $\%$. If we choose to minimize dishing without violating the concentration criterion $(C \geq 2 \mathrm{wt} . \%)$, the procedure to obtain the settings of variables $(P, V$, and $C)$ is even simpler. 


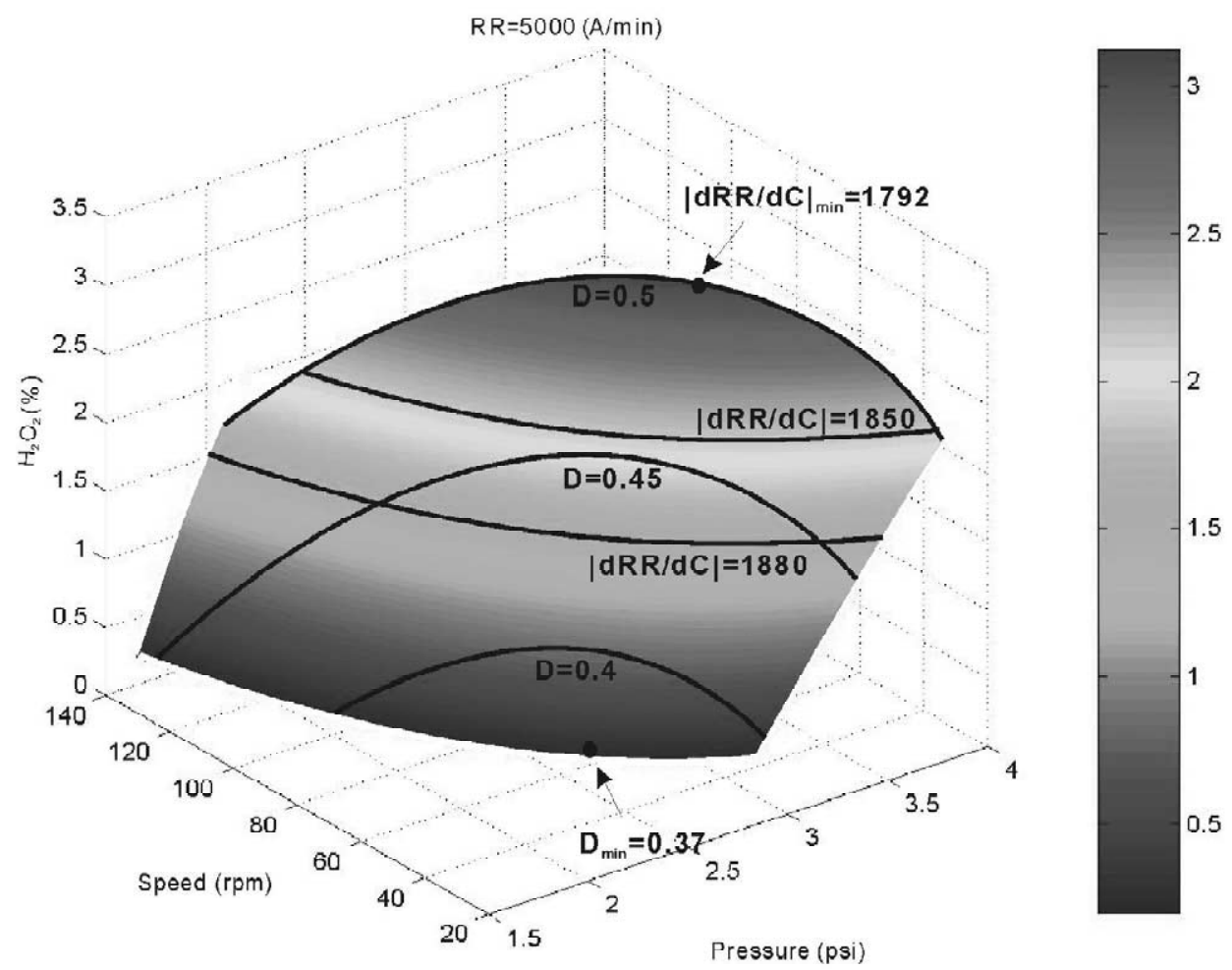

Fig. 7. Feasible operation region $(D \leq 0.5$ and $S \leq 2000)$ and constant dishing and sensitivity contours for RR=2000 A $/$ min.

1. Set the oxidizer concentration to the smallest acceptable value (e.g. $C=2$ wt.\%).

2. Set the sensitivity $(S)$ to the largest acceptable value in the feasible region.

3. Solve Eqs. (9) and (11) simultaneously for $P$ and $V$.

The reason is quite obvious: the optimal operation conditions always lie on the constraints as shown in Figs. 6 and 7. Following the procedure, the results (4th column in Tables 2 and 3) show that dishings

Table 2

Optimized operation condition for $\mathrm{RR}=2000 \AA \mathrm{A} / \mathrm{min}$

\begin{tabular}{llll}
\hline & $\begin{array}{l}\text { Minimizing } \\
\text { dishing }\end{array}$ & $\begin{array}{l}\text { Minimizing } \\
\text { sensitivity }\end{array}$ & $\begin{array}{l}\text { Minimizing dishing } \\
\text { and constraint on } \\
\mathrm{H}_{2} \mathrm{O}_{2} \text { conc. }(\geq 2 \%)\end{array}$ \\
\hline $\mathrm{H}_{2} \mathrm{O}_{2}$ Conc (\%) & 0.08 & 5.9 & 2.0 \\
Pressure (p.s.i.) & 1.8 & 3.2 & 2.2 \\
Speed (rpm) & 45 & 75 & 56 \\
Dishing & 0.28 & 0.5 & 0.35 \\
Sensitivity $|\mathrm{dRR} / \mathrm{d} C|$ & 771 & 586 & 738 \\
\hline
\end{tabular}


Table 3

Optimized operation condition for $\mathrm{RR}=5000 \AA \mathrm{A} / \mathrm{min}$

\begin{tabular}{llll}
\hline & $\begin{array}{l}\text { Minimizing } \\
\text { dishing }\end{array}$ & $\begin{array}{l}\text { Minimizing } \\
\text { sensitivity }\end{array}$ & $\begin{array}{l}\text { Minimizing dishing } \\
\text { and constraint on } \\
\mathrm{H}_{2} \mathrm{O}_{2} \text { conc. }(\geq 2 \%)\end{array}$ \\
\hline $\mathrm{H}_{2} \mathrm{O}_{2}$ Conc. (\%) & 0.2 & 3.1 & 2.0 \\
Pressure (p.s.i.) & 2.4 & 3.2 & 2.8 \\
Speed (rpm) & 58 & 75 & 72 \\
Dishing & 0.37 & 0.5 & 0.45 \\
Sensitivity $|\mathrm{dRR} / \mathrm{d} C|$ & 1928 & 1792 & 1857 \\
\hline
\end{tabular}

are 0.35 and 0.45 for the removal rates of 2000 and $5000 \AA$ /min, respectively. This is the polisher setting recommended to maintain a robust operation.

\subsection{Overall strategy}

Up to this point, we have discussed the case where a fixed $R R$ (e.g. $R R=5000 \AA / m i n$ ) is applied throughout the entire polishing process. In practice different strategies can be implemented to achieve
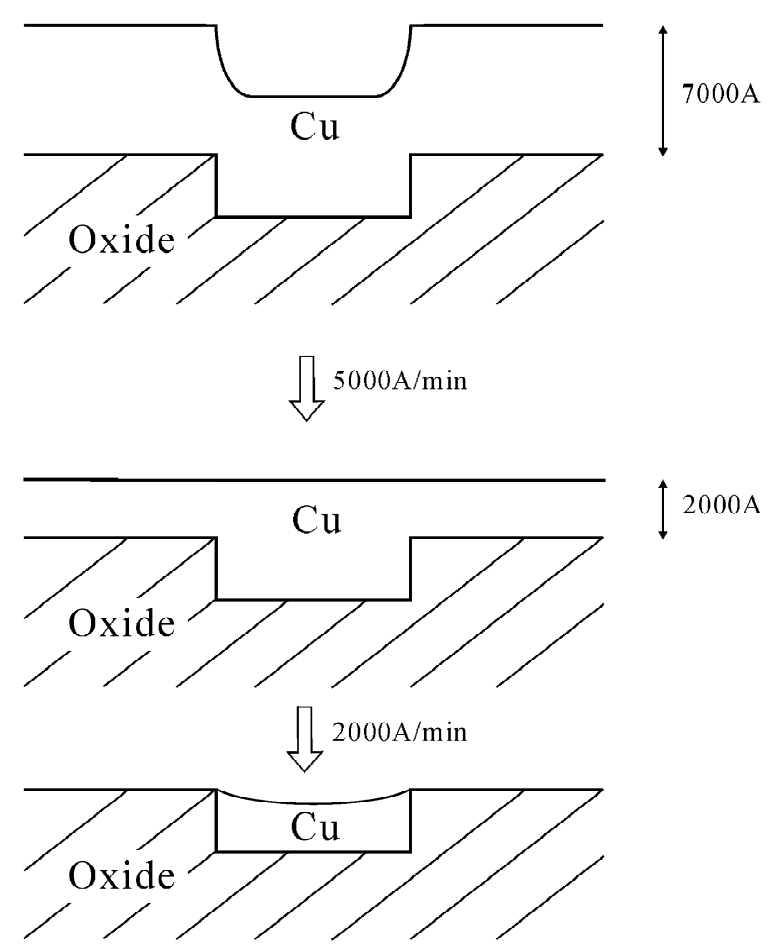

Fig. 8. Conceptual description of the soft landing ( $R R=5000 \AA / m i n$ for the first $5000 \AA$ and $\mathrm{RR}=2000 \AA / \mathrm{min}$ for the remaining $2000 \AA$ A). 
a higher throughput while maintaining stable operation (e.g. acceptable dishing). One common strategy is the 'soft landing' where a high RR is employed first, for high throughput, and when the end-point is approaching, a smaller RR follows, for smaller dishing. Fig. 8 shows the concept of soft landing. The following example illustrates how to incorporate the proposed robust operation into the soft landing strategy.

The total thickness to be removed is $7000 \AA$ and two different removal rates, $2000 \AA / \mathrm{min}$ and 5000 $\AA /$ min, can be applied. The pressure and speed can be adjusted at different stages, but the oxidizer concentration remains the same (we can change the flow rate of the slurry but not the composition during a CMP cycle). Again, all the constraints are applied to the $\mathrm{Cu}$ CMP. First, at the low RR (2000 $\AA / \mathrm{min})$, we set the oxidizer concentration to the smallest acceptable value, $C=2 \mathrm{wt} . \%$, and compute the $P$ and $V$ such that dishing is minimized (following the procedure in the previous section). The result is exactly the same as the recommended settings (Table 2). At the high RR stage (5000 $\AA / \mathrm{min})$, we proceed to find $P$ and $V$, again, by minimizing dishing and the result is shown in the 4th column of Table 3. Note that another reason for minimizing dishing is that it gives a smaller operation pressure which can alleviate surface damage caused by possible pad defects. Table 4 summarizes settings of operation variables for three different strategies: high RR, low RR, and soft landing. The case of high RR results in the shortest polishing time $(1.4 \mathrm{~min})$ with a large dishing $(D=0.45)$ and, on the contrary, the low RR strategy gives a longer polishing time $(3.5 \mathrm{~min})$ with a smaller dishing $(D=0.35)$. The strategy of soft landing leads to an acceptable dishing $(D=0.35)$ while maintaining a relatively efficient operation (polish time $=2 \mathrm{~min}$, a $40 \%$ reduction compared to low RR). Moreover, this can be achieved simply by adjusting settings of existing polishers.

\subsection{Summary}

The results presented here are derived from a commercial polisher with a specific slurry, but, without loss of generality the procedure can be extended to different $\mathrm{Cu} C M P$ with $\mathrm{H}_{2} \mathrm{O}_{2}$ as oxidizer. For a given polisher and slurry, the procedure consists of the following steps.

1. Perform at least one set of experiments to obtain the relationship between $R R$ and oxidizer concentrations (e.g. Fig. 1).

Table 4

Different operation policies and resulting dishing, sensitivity and total polishing time

\begin{tabular}{|c|c|c|c|}
\hline & $\begin{array}{l}\text { Strategy I } \\
\text { (High RR) }\end{array}$ & $\begin{array}{l}\text { Strategy II } \\
\text { (Low RR) }\end{array}$ & $\begin{array}{l}\text { Strategy III } \\
\text { (Soft landing) }\end{array}$ \\
\hline RR (first $5000 \AA$ A) & 5000 & 2000 & 5000 \\
\hline RR (remaining $2000 \AA$ ) & 5000 & 2000 & 2000 \\
\hline $\mathrm{H}_{2} \mathrm{O}_{2}$ Conc. $(\%)$ & 2.0 & 2.0 & 2.0 \\
\hline Pressure (p.s.i.) & 2.8 & 2.2 & $2.8 / 2.2$ \\
\hline Speed (rpm) & 72 & 56 & $72 / 56$ \\
\hline Dishing & 0.45 & 0.35 & 0.35 \\
\hline Sensitivity $(|\mathrm{dRR} / \mathrm{d} C|)$ & 1857 & 738 & $1857 / 738$ \\
\hline Polishing time (min) & 1.4 & 3.5 & 2 \\
\hline
\end{tabular}


2. Construct the modified Preston equation (Eq. (9)) from regression of the experimental data. Note that the exponent of the pressure can be re-calculated if more than one data sets are available.

3. Apply the dishing model (Eq. (11)). If data are available, the multilinear model can be re-parameterized.

4. Implement the soft landing strategy (Fig. 8).

a At the low RR stage (the 2nd stage in Fig. 8), set the oxidizer concentration to the smallest acceptable value (e.g. $C=2 \mathrm{wt} . \%$ ), and compute the $P$ and $V$ such that the dishing is minimized (Eq. (9)) while satisfying all possible constraints (e.g. sensitivity of Eq. (10), high and/or low limits for pressure and speed).

b For the high RR stage (the 1st stage in Fig. 8), repeat the previous step except that the oxidizer is already fixed at the smallest acceptable value.

The procedure is useful in achieving a stable CMP operation while maintaining high throughput. More importantly, this can be done by performing simple experiments on existing polisher and constructing operation-relevant models accordingly.

\section{Conclusions}

In this work, the potential problem, non-monotonic oxidizer concentration effect, in $\mathrm{Cu} C \mathrm{CP}$ is pointed out and verified experimentally. This complicates already difficult operation problems. Two operation-relevant models are proposed to ensure a robust operation. The first model adds the concentration effect into the well-known Preston equation and model parameters can be obtained by performing simple regression from experimental data. Next, a multilinear model is proposed to describe the effect of operation variables on dishing. Combining both models, we are able to locate a feasible operation region without constraint violation (e.g. dishing and sensitivity with respect to concentration variation). Furthermore, optimized operation conditions, on dishing or sensitivity, can be computed analytically. The optimal operation conditions are incorporated in a polishing strategy, soft landing. The results (e.g. Table 4) clearly indicate that we can achieve a stable operation while maintaining a relatively high throughput. Finally, a systematic procedure, from performing experiments to finding the settings, is proposed to ensure a robust operation.

\section{Acknowledgements}

Y.C.K. and C.C.Y. are grateful for the financial support of the National Science Council of Taiwan.

\section{References}

[1] C.Y. Chen, C.C. Yu, S.H. Shen, M. Ho, Operational aspects of chemical mechanical polishing: Polish pad profile optimization, J. Electrochem. Soc. 147 (2000) 3922.

[2] L.M. Cook, Chemical process in glass polishing, J. Non-cryst. Solids 120 (1990) 152. 
[3] F.B. Kaufman, D.B. Thompson, M.A. Jaso, W.L. Guthrie, D.J. Pearson, M.B. Small, Chemical-mechanical polishing for fabricating patterned W metal features as chip interconnects, J. Electrochem. Soc. 138 (1991) 3460.

[4] C.W. Liu, B.T. Dai, C.F. Yeh, Modeling of the wear mechanism during chemical mechanical polishing, J. Electrochem. Soc. 143 (1996) 716.

[5] V. Nguyen, H. VanKranenburg, P. Woerlee, Dependency of dishing on polish time and slurry chemistry in Cu CMP, Microelectron. Eng. 50 (2000) 403.

[6] F. Preston, The traction of glass polishing, J. Soc. Glass Technol. 11 (1927) 214.

[7] J.M. Steigerwald, S.P. Murarka, R.J. Gutmann, Chemical Mechanical Planarization of Microelectronic Materials, John Wiley and Sons, New York, 1997.

[8] Y.L. Wang, J. Wu, C.W. Liu, T.C. Wang, J. Dun, Material characteristics and chemical mechanical polishing of aluminum alloy thin films, Thin Solid Films 332 (1998) 397.

[9] K. Wijekoon, S. Tsai, M. Chandrachood, B. Brown, F. Redeker, S. Nanjangud, G. Amico, Multi-Step Approach to Copper CMP Development, SEMI/Japan Technical Symposium (1998).

[10] D. Zeidler, Z. Stavreva, M. Plotner, K. Drescher, Characterization of $\mathrm{Cu}$ chemical mechanical polishing by electrochemical investigations, Microelectron. Eng. 33 (1997) 259. 